\title{
SAISIE ET ANALYSE DE DONNÉES DES COURANTS PARTICULAIRES ET PRESSIONS AU VOISINAGE DU FOND DANS LA HOULE LITTORALE PROCHE DU DÉFERLEMENT *
}

par

M. ARHAN

et

R. EZRATY

Equipe d'Océanographie physique Centre Océanologlque de Bretagne Brest

Nous continuons, pour cet article, la présentation permettant une publication rapide d'articles présentant une partie mathématique importante

LA HOUILLE BLANCHE 
Il est de fait que la houle côtière à la limite du défer lement est redoutée par l'Ingénieur lors de l'évaluation des efforts qui s'exerçen sur les ouvrages de Cénie Civil à la côte. Ceci est du à la nature complexe du phênomène que lês modèles théoriques ne peuvent actuellement représenter qu'en partie.

Sur le plan expérimental, les moyens d'observation dont nous disposons permettent d'étudier quantitativement la houle côtięre proche du déferlement, de mettre en évidence certaines de ses propriétés, de tester cer tains modèles théoriques valables au large et qu'il est possible d'adapter à la côte.

Cette étude met en évidence la forte non-1 inéarité des déplacenents de la surface libre, elle prếcise les conditions d'utilisation des théories d'AIRY et de l'onde longue pour l'appréciation des courants particulaires, enfin elle présente un modèle de la répartition statistique des maximums de la surface libre qui gêneêralise les résultats bien connus de CARTKR IGHT et L.ONGUET-HIGG INGS valables en eau profonde.
I. DLSPOSITIF EXPER LMENTAL - PRETRAITEMYNT DES SIGNAUX - EXPLOITATION DES ERREGISTREMENTS.

1. Dispositif expérimental

L'Equipe d'Océanographie Physique du Centre océanologique de Bretagne a étudié et implanté une station de mesure de houle littorales sur la pląe de la palue, sicuée dans la prêsqu'île de crozon. Le site a été choisi en raison de sa faible pente $(2,5 \%)$ et de son exposition aux fortes houles d' 'Uuest arrivant de 1 'At lantique.

Les mesures exploitêes dans cette étude proviennent de deux campagnes effectuées en janvier et mai 1974. iors de la première opération des mesures simultanées de pression au fond et de vitesses horizontales ont été réalisées par des fonds $n$ 'excédant pas 4 mètres. La deuxième opération menée plus au large, par des fond́s de 10 mècres, a permis d'enregistrer les déplacements de la surface libre (Fig. 1), Ráf. [1] et [4]

Le courantomètre à đcux voies BEN-COMEX utilisế est du type êlectromagnétique L'étalonne indique une erreur absolue

inférieure à $5 \mathrm{~cm} / \mathrm{s}$ sur $0-20 \mathrm{~cm} / \mathrm{s}$ et une précicion de $2 \%$ sur $0,20-3 \mathrm{~m} / \mathrm{s}$.

Le capteur de pression absolue est posé au fond, sa précision est estimée à $10 \mathrm{~cm} \mathrm{~d}$ 'eau sur la pression moyenne enregistrée.

L.a bouće Datawe11 moni11lée plus au large intègre les signaux d'u accêléromètre stabilisé verticalement. Sa bande passante cst limitée à $0,06-0,8 \mathrm{~Hz}$

\section{Prêtraitement des signaux}

La composante de marée est éliminée sur les signaux de pression et de courants particulaires à l'a ide d'un filtre passe-haut constitué par une régression linéaire sur la durée de l'cenregistroment.

Le traré analogique du signal en provenance de la bouce natawell prêsente des fréquences supérieures à $0.8 \mathrm{kz}$, thucs ia des defauts de lä liaison radio et à des oscillations parasites de la lane cansíes par le vent. Puou éliminer ces parasites, le signal est traitê par un filtre passé-bas symćtrique, affectant les poids $1 / 4-1 / 2-1 / 4$ à 3 points consécut ifs échantilIonnës 4 fois par seconde. Ce filltre a pour cocfricient d'artánualion 
$R(\hat{f})=(1+\cos 2 \pi f \delta) / 2$

oũ $\delta$ est la valeur du pas d'écha:tillionage (ici 0,25 s).

La vitesse horizontale au sein du fluide est connue à chaque instant par ses composantes suivant les axes du courantomètre. La position du courantomètre étant indépendante de la direction de la houle, un nouveau repère est défini pour chaquue enregistrement, tel que les deux composantes de la vitesse soient non corrélées. Celui des deux axes correspondant au plus grand transport d'énergie est considéré comne 1 'axe moyen de propagation de la houle. Les traitements statistiques décríts ci-après ont été effectués sur les données rapportées à ce nouveau système d'axe, dont la figure 2 présente un échantillon.

\section{Exploitation des enregistrements}

Un traitement statistique classique, comprenant la dêtermination de l'histogramme et le calcul de la densitế spectrale, a été effectué sur chaque signal. La détermination des coefficients de corrélation et les cohérences entre variationsde pression au fond et courants particulaires a permis de préciser la nature des relations entre ces deux variables. Les données de niveau (pression au fond et signal de la bouée Datawel1) ont áté soumises à une analyse " vague par vague "afin de tester la validité de la loi de probabilité de RAYLEIGH pour les hauteurs " crête à creux " en eau peu profonde.

II. ANAT.YSE DES DONREES ET COMPARAISON DES RESULTATS AUX MODELES THEORIDUES

\section{Non-1 inéaritế des signaux}

Sur les figures 3 (a) et 4 (a) sont tracés les histogrammes des variables centrées normées pet u (pression au fond et vitesse dars le sens de la houle), ainsi que la courbe de Gallss de variance unite ces distributions s'écartent sensiblement de la loi normale. Le calcul du moment d'ordre 3 de la distribution (facteur de dissynétrie) et du moment d'ordre 4 (facteur d'applatissempnt) permet de chiffrer ces écarts. Si les coeffi-

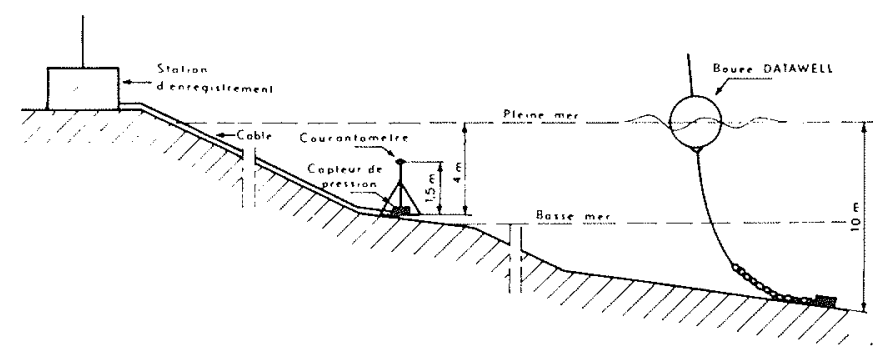

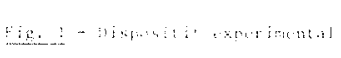

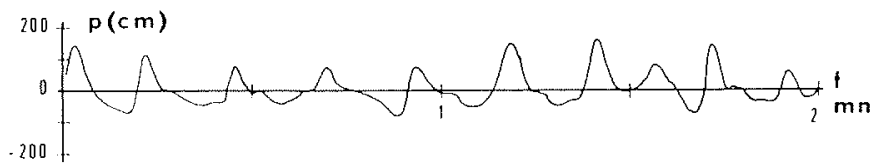

$200+u(\mathrm{~cm} / \mathrm{s})$

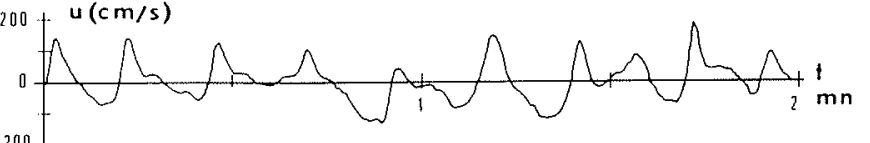
200

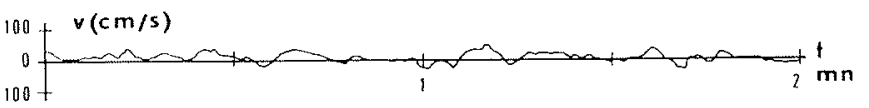




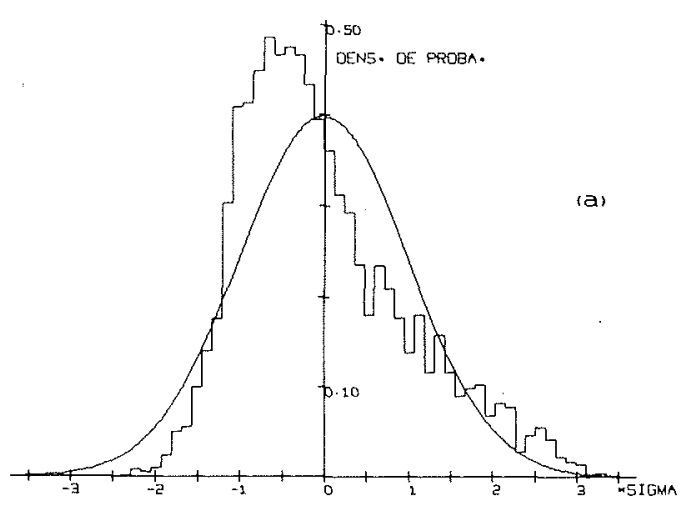

ENR. NO. 2 PRESSION AU FONO

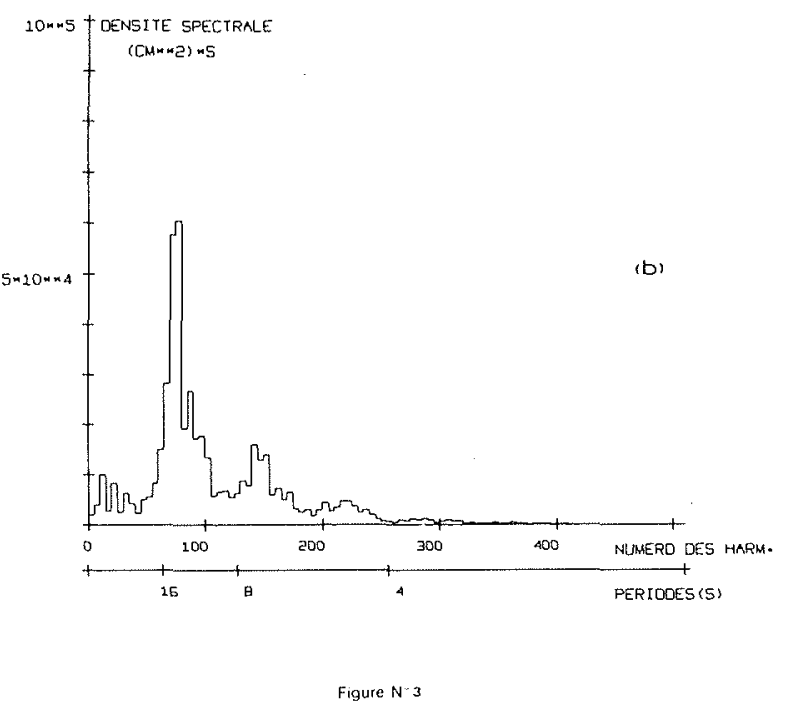

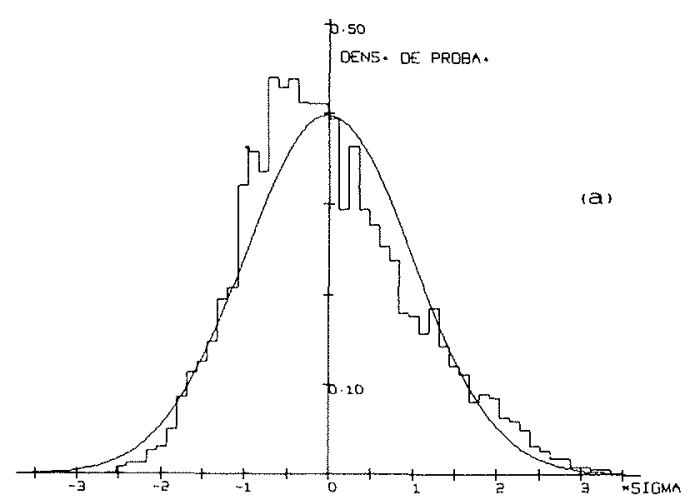

ENR. NO. 2 VITESSE DS LE SENS DE LA hOULE

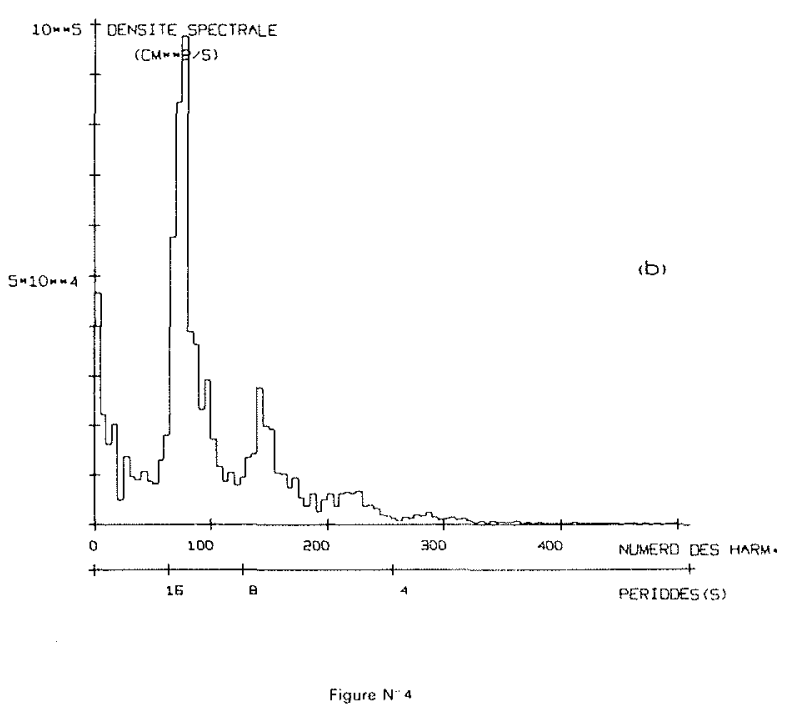


cients d'applatissement rencontrés au cours des 22 enregistrements effectués $s^{\prime}$ écartent peu de la valeur gaussienne, et sont centrés autour de celle-ci

$\left(m_{4}=3\right)$, les facteurs de dissymétrie différent considérablement de la valeur de la loi normale $\left(m_{3}=0\right)$.

La figure $5(b)$ prêsente les fonctions de répartition des coefficicients de dissynetrie observés dans la zone de déferlement (mesure de pression) et plus au large (bouée DẠTALELL). Les valeurs rencontrées par $10 \mathrm{~m}$ de fond sont du même ordre de grandeur que celles fournies par KINSMAN [6] et LONGUETHIGGINGS [8] et dans ce cas, la distribution peut être représentée par une loi de GRAM-CHARLIER. Les valeurs élevées du coefficient de dissymétrie par 4 m de fond indiquent une non-1 inéarité beaucoup plus importante se produisant lorsque 1 'onde aborde la zone de déferlement. Deux histogramnes caractéristiques reportés sur le dême graphique (Fig.5(a)) permettent de mieux visualiser les dëformations de la surface libre à 1 'approche du rivage. La forme de 1 'histograne des variations de pression traduit l'applatissement du creax đu défer lement.

Les spectres d'énergie sont présentês sur les figures 3 (b) et $4(\mathrm{~b})$ 1 a prëcision de l'estimation de ces spectres. Pour des signaux gaussiens chaque niveau d'éncrgic aurait une répartition en $X^{2}$ à 20 degrés de liberté. Sur certains spectres (Fig. 3(b)) dés maxirums secondaires existent aux harmoniques de la période fondamentale de la houle. A l'approche du rivage, les conditions non-1inéaires satisfaites à la surface libra produisent un transfert d'énergie de la fréquence fondamentale de la houle au large vers les fréquences multiples de celle-cí. Ces résul tats expérimentaux sont à rapprocher du modèle théorique de houle non-1inéaire, êtabli par L. TrCK [13] qui met en évidence de tels maximums secondaires du spectre.

2. Corrélation entre les variacions de pression au fond et la vitesse dans 10 . direction de la houle.

a) Rappels thêoriques

Les variations de pression au fond autour de la valeur moyenne sont notées $\mathrm{p}$, les pressions totales $\mathrm{P}$ et la pression moyenne $\mathrm{P}_{\mathrm{n}}$. - Theorie linéaire d'AIRY : dans les conditions de l'expérience ct pour une onde monochromatique de longueur $\mathrm{L}=2 \pi / \mathrm{k}$ et de pulsation ", on a

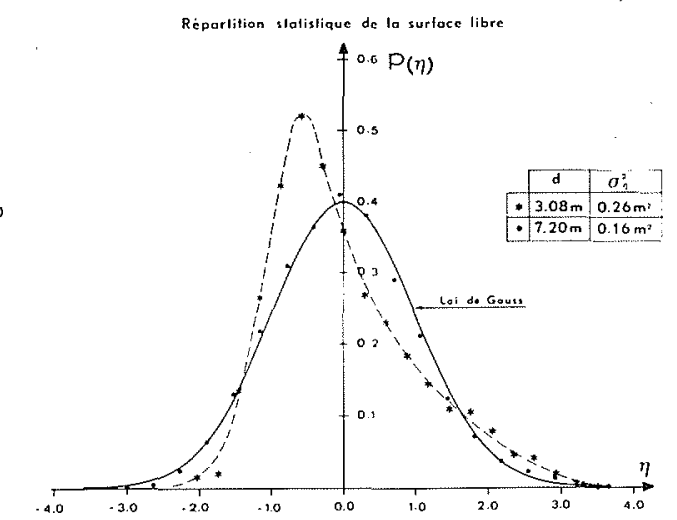

(b)

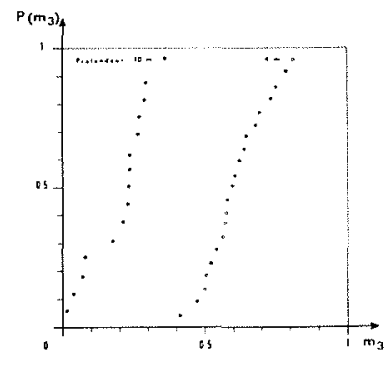

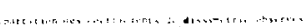

Figure $\mathrm{N}^{\circ} \mathrm{s}$ 


$$
p=\frac{p_{0}}{c_{1 x k h_{0}}} u
$$

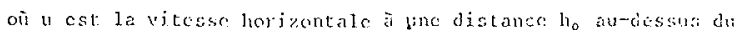

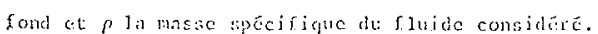

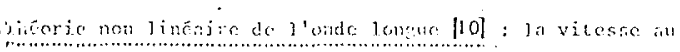
scin du [misle est const tinte diuns uin plas vertical perpentica-

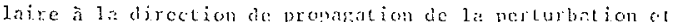
pour valcur

$$
u=2 \sqrt{i}-2 \sqrt{i n}
$$

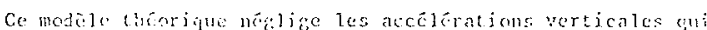

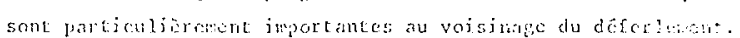
b) Coiminition:

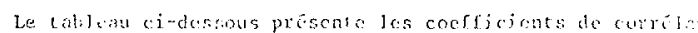

\begin{tabular}{|c|c|c|c|c|}
\hline at & 2 & 5 & 6 & 7 \\
\hline 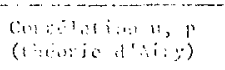 & 0.203 & $0,8, ?$ & 0,872 & 0,336 \\
\hline (and & $0, \ldots \ldots$ & 0,5772 & 0,636 & $0,8:, 5:$ \\
\hline
\end{tabular}

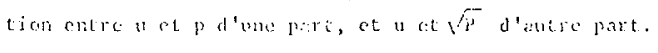

Il n'est pas possible, au vu de ces resultats, de faire un choix entre les deus: theories. Les mates de points $(u, p)$ o $(u, \sqrt{8})$ présentics sur los figures $6(3)$ at $7(a)$, ont des fornes trick voisines. tha figura $6(a)$ perte aussi l'are de parabole d'equntion

$$
\mathrm{u}=2 \sqrt{\frac{1}{p}}-2 \sqrt{\frac{\mathrm{xt}}{p}}
$$

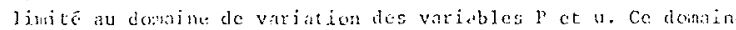

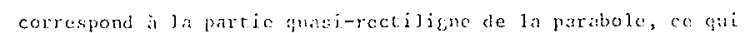

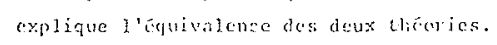

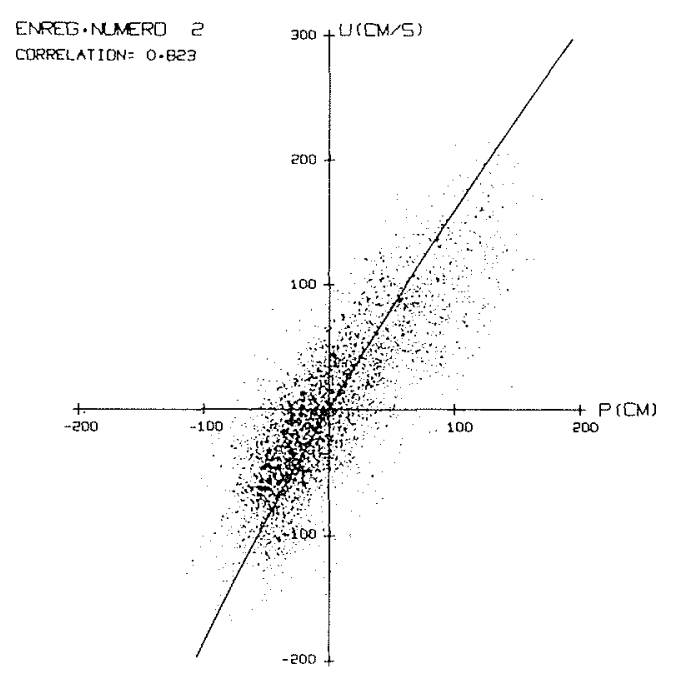

ENSEMBLE DES POINTS $(U, P)$

(b)

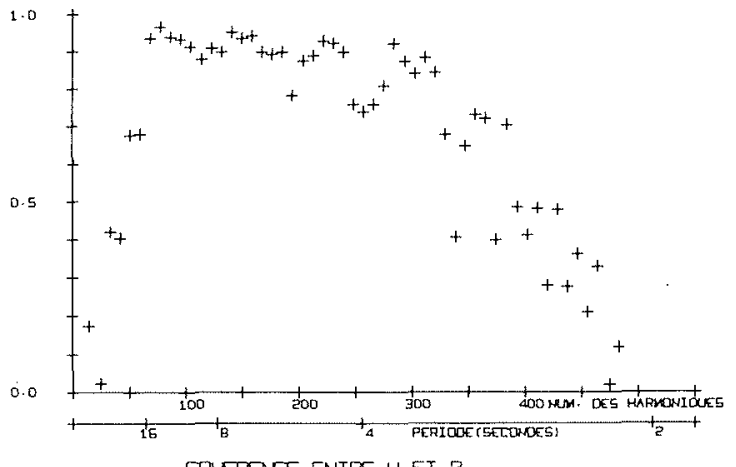

COHERENCE ENTRE U ETP 


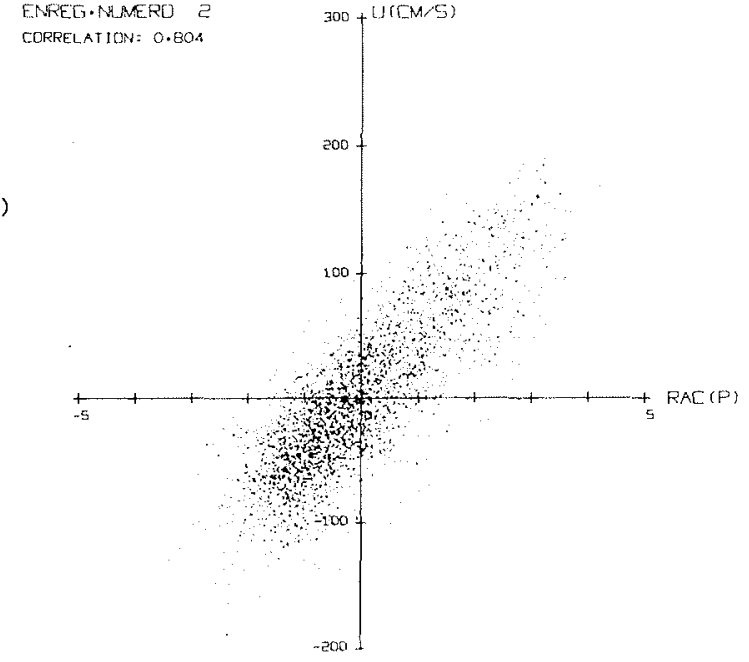

ENSEMELE [FE FLINTS (U,RACTP)

(b)

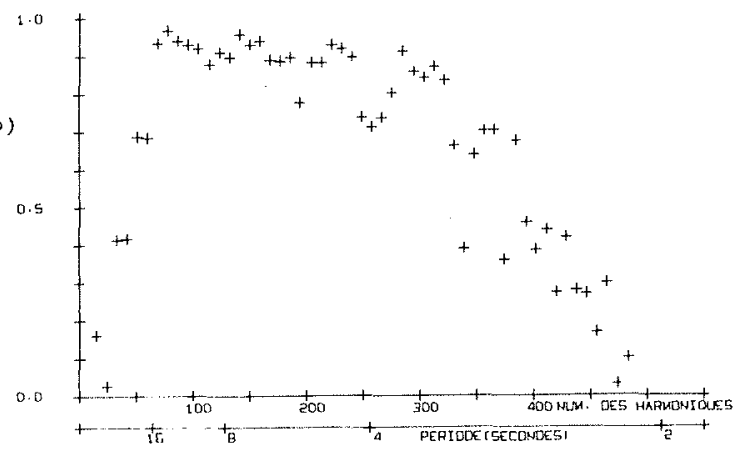

C.CHEREMNE ENTRE U ET RACIP]

Eigure $N^{\prime} 7$
La pente moyenne des nuages de points est de 1 'ordre de $u / p=1,5$. Les pentes théoriques seraient 1,82 pour une houle d'ATRY de période $6 \mathrm{~s}$ et 1,76 pour une houle d'AIRY de période $15 \mathrm{~s}$. Pour les très grandes périodes, les deux théories fournissent le même résultat

$$
\mathrm{u}=\frac{\mathrm{p}}{\sqrt{\sigma \mathrm{P}_{\mathrm{p}}}}=1,72 \mathrm{p} .
$$

La dispersion des points expérimentaux autour des courbes théoriques dans ces nuages de points est due à plusieurs causes :

- la houle enregistrée n'cst pas monochromatique et le rapport théorique u/p dépend de la période,

- le courantomètre, plus proche de la surface libre que 1e capteur de pression, décèle des petites oscillations que celui-ci ignore,

- seule la composante de la vicesse dans le seris de la propagatio moyenne de la houle est prise en compte, tandis que p résulte de la superposition de plusieurs ondes se propageant dans des directions différentes,

- par aillcurs, l'étude des spectres de courants revèle l'existence de phénomènes étrangers à la houle (courants côtiers de périndes de 4 à 5 minutes). Le calcul des cohérences entre signaux pormet de prếciser 1 e doma ine de frëquences intéressé par ces phénonènes.

c) Cohêr ences

Les cohérences sont représentées sur les figures $6(\mathrm{~b})$ et $7(\mathrm{~b})$ pour les couples de variables $(u, p)$ et $(u, \sqrt{P})$. Les deux tracés sont très voisins en raison de l'équivalence des deux thêories expliquéc dans le parayraphe précédent. Trois domaines de périodes distincts ressortent de ces courbes :

- pour les composantes du spectre ayant des périodes comprises

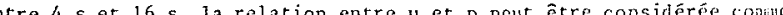
Linéaire (cohérence supérieure à 0,9 ). Dans ce domaine la houle cost le seul signal enregistré

$$
\text { - pour les pêriodes supérieures à } 16 \text { secondes, la chute }
$$


de cohérence traduit 1 'existence de courants côtiers de longues périodes : que par :

- Ia décroissance de cohérence au-dessous de 4 secondes. s'expli-

- Le filtrage hydrostatique du à la différence de niveau entre courantomètre et capteur de pression.

- L'apparition, pour ces hautes fréquences, de la turbulence, particulièrement sensible dans la zone de mesure située à la limite du déferlement.

\section{Modèle de répartition des hauteurs de vagues}

a) Répartition des hauteurs de vagues hors de la zone de déferlement.

LONGUET-HTGGINGS [7] puis CARTWRTGHT et LONGUET-HIGG INGS [3] ont montré que la répartition des hauteurs de vagues, h, suit une loi de RAYLEIGH si 1 'on suppose que les dếplacements de la surface libre résultent d'une superposition linéaire d'ondes élémentaires de phase aléatoire et de fréquences voisines constituant un spectre " étroit ". La densité de probabilité des hauteurs, $p(h)$, s'écrit alors :

$$
p(h)=\frac{2 h}{a^{2}} e^{-\frac{h^{2}}{a^{2}}}
$$

on en déduit la moyenne $\overline{\mathrm{H}}=\mathrm{a} \frac{\sqrt{\pi}}{2}$ et la variance $\sigma_{\mathrm{h}}^{2}=\mathrm{a}^{2}\left(1-\frac{\pi}{4}\right)$ les hypothèses précédentes permettent de déduir e:a $=2 \sqrt{2} \sigma_{y}$ $\sigma_{\eta}$ étant l'écart type du niveau de la surface $1 \mathrm{ibre}$.

pour comparer les fonctions de répartition theooriques et expérimentales il est comnode d'utiliser un système d'axes gradué d'une part suivant la loi de probabilité et d'autre part en hautcur rapportće au paramètre de la loi de RAYLEIGH.

On constate sur la figure 8 que $1 \mathrm{cs}$ mesures erfectuées par $10 \mathrm{~m}$ d'eau cožncident avec celles de l'ouragan carla par 30 mètres d'eźu.

La Loi de RAYBEIGH, basće sur 1 'hyputhöse d'un spectre étroit sous-estinle le nombre de vagues de petite amplitude. Ella s avicre satis-

\section{Répartilion statistique des hauteurs de vagues au large}

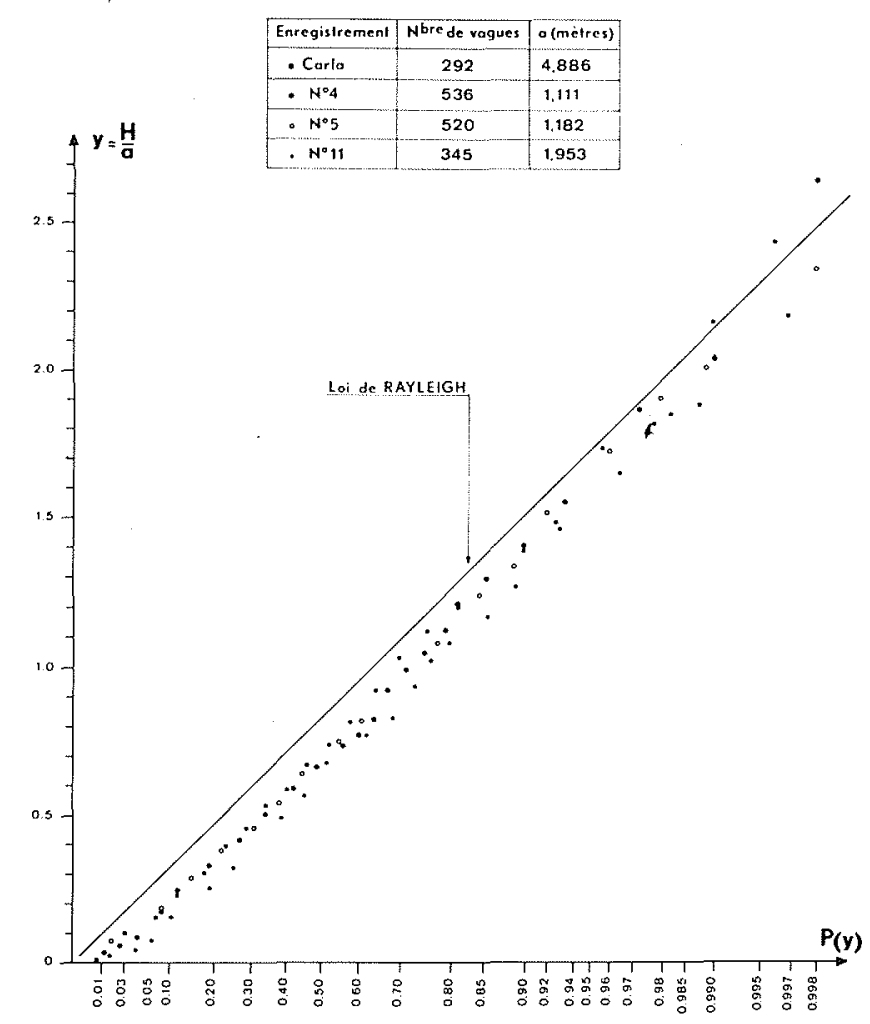

Figure N's 
faisante pour les grandes vagues lorsque 1 'on utilise la variance expérimentale $o_{\mathrm{h}}^{2}$ pour calculer le paramètre caractéristique " a ".

b) Répartition des hauteurs de vagues par faible profondeur

Lor sque la hauteur de vague est du même ordre de grandcur que la profondeur d'eau, coome c'est le cas à la côte dans la zone de déferlement, il est plus représentatif d'un état de mer d'utiliser, au lieu de la hauteur $h$ de la vague, le creux réduit " $h_{r}$ ", rapport de la hauteur de la vague à la profondeur moyenne à cet instant.

On constate alors (Fig.g) que la loi de probabilité du creux réduit s'écarte notablement de la loi de RAYLEIGH (THOMSON [1] ). A l'encontre des observations par 10 mètres d'èau, cette distribution prédit, dans la zone proche du déferlement, un plus grand nombre de vagues de petite amplitude et un plus petit nombre de vagues de forte amplitude que l'expérience.

Par ailleurs, la forte concavité des courbes expérimentales suggère une tendance asymptotique horizontale pour les vagues de forte amplitude. Cette allure de courbe s'explique physiquement : pour une hauteur d'eau donnée, il n'est pas possible de rencontrer des vagues de hauteur supérie'lže à la profondeur. Les valeurs des grandes amplitudes sont limitées, ce qui diminue fortement la variance de $h_{r}$ et par consé

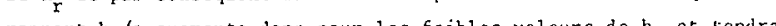
rapport $h_{r}$ /a augmente donc pour les faibles valeurs de $h_{r}$ et tendrait vers $1 /$ a pour toutes les vagues de hauteur égale à la profondcur d'eau, c'est à dire à saturation.

c) Rappe1 de la loi de WeIBULL

La probabilité cumulative $\mathrm{P}(\mathrm{x})$ de la loi de [N:IL.BltL., est do la
Répartition statistique des hauteurs de vagues

par faible profondeur

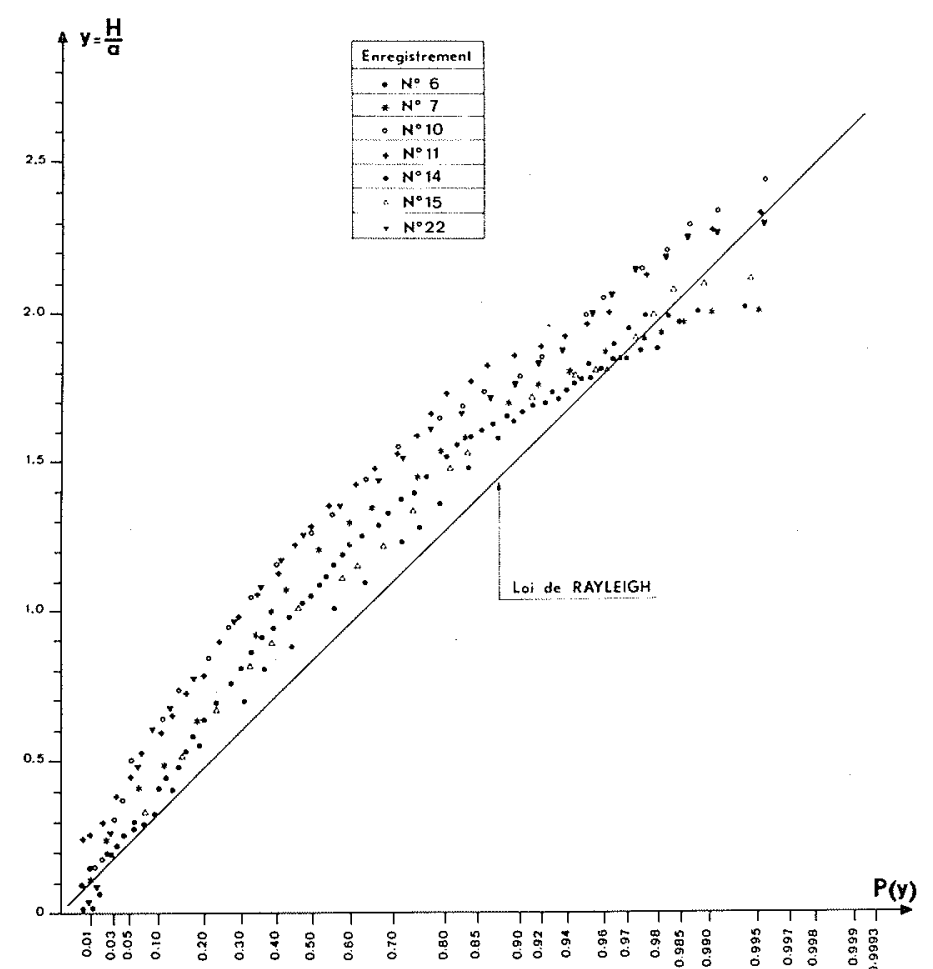

Eigure $N^{n} 9$ 
fr: pe :

$$
P(x)=1-e^{-\left(\frac{x-\epsilon}{a-\epsilon}\right)^{c}} \quad \text { pour } x \geqslant \epsilon
$$

où $\varepsilon$ est la valeur minimum de la variable $x$,

a est le paramètre caractéristique de la loi,

c est le paramètre de forme.

On remarque immédiatenent qu'une loi de hEIBùĭ se réduit, avec $\epsilon=0$ et $\mathrm{c}=2$, à une loi de RAYLETGH.

Utilisant la fonction densité de probabilité

$$
P(x)=\frac{c}{(a-c)}\left(\frac{x-c}{a-c}\right)^{c-1} e^{-\left(\frac{x-\epsilon}{a-c}\right)^{c}}
$$

i1 vient avec

Il faut noter que la courbe de densité de probabilité devient de plus en plus étroite au fur et à mesure que "c" augrente (Fig-10) Ceci s'interprête comme une accumulation de plus en plus important des valeurs de $x$ autour de sa valeur la plus probable lorsçue "c" crồt.

Lorsque l'on représente la loi de probabilitê du creux relatif des vagues par une $10 \mathrm{i}$ de HEIBULh, le paramètre $c$, correspondant à la yaleur ininimum de hest, évidement, nul. Il est possible de calculer à l'aide des relations précédiantes (moycune et variance) les valeurs de "a " ct " c " ; núarmoins ure meilicure cstimation de ces paramètre peut être oblenue par dẹternilnation graphique ce qui permet de prendre en compte le comportement globial de l' tencegiscrement.

La fonction de répartition d'une loi de wesliult s'écrit

Densité de probabilifé

Loi de Weibul

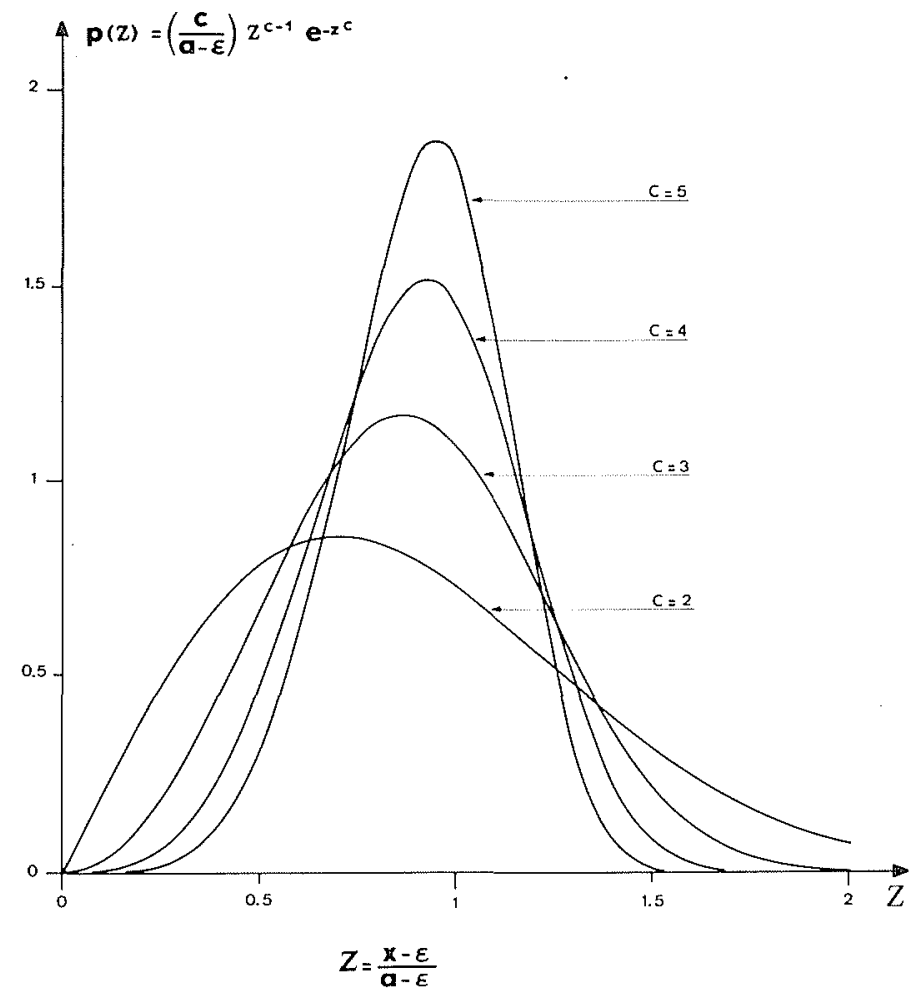

Figure $\mathrm{N} 10$ 


$$
P(x)=1-e^{-\left(\frac{h}{a}\right)^{c}}
$$

Il en découle:

$$
\operatorname{Ln}[-\operatorname{Ln}(1-P(x))]=c \operatorname{Ln}(x)-c \operatorname{Ln}(a)
$$

sur un graphique orthonormé " $c$ " apparaît comme la pente d'une droite d'ordonnée à 1 'origine $-c \ln (a)$.

d) Estimation de la saturation

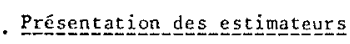

La hauteur d'une vague, de cambrure inférieure à la cambrure 1imite, ne peut guère dépasser la profondeur d'eau ; 1e creux relátíf sera donc au plus égal à. 1 .

Un échantillon expérimental sera d'autant plus saturé que le nombre de creux relatifs voisirs de 1 sera élevé ; les grandeurs $\mathrm{H}(1 / 10), \mathrm{H}(1 / 3) \overline{\mathrm{H}}$ ainsi que les rapports $\mathrm{H}(1 / 3) / \mathrm{H}(1 / 10) \mathrm{e}=\overline{\mathrm{H}} / \mathrm{H}(1 / 10)$ tendront alors vers 1.Le rapporH $(1 / 3) / \mathrm{H}(1 / 10)$ sera significatif de 12 saturation des plus hautes vagues, alors que $\vec{H} / H(1 / 10)$ indiquera le comportement global de l'échantillon. Il faut néanmoins noter que $\overline{\mathrm{H}}$ étant fortement sensible au grand nombre de petites vagues, le rapport $\overline{\mathrm{H}} / \mathrm{H}(1 / 10)$ sera expérimentalement minoré par rapport au cas du spectre étroit.

Enfin, ces rapports sont uniquenent fonction du paramètre dc forme "c"; en effet, si l'on note par h le creux relatif,

$P(h)=1-e^{-\left(\frac{h}{a}\right)^{c}}$

(1)

et $\quad p(h)=\frac{c}{a}\left(\frac{h}{a}\right)^{c-1} e^{-\left(\frac{h}{a}\right)^{c}}$

par définition :

ห $1 / n=\int_{h_{0}}^{\infty} h p(h) d h / \int_{h_{0}}^{\infty} p(h) d h$
La borne inférieure d'intégration tho est dêterminée par la relation

$$
P\left(h_{0}\right)=1-\frac{1}{n},
$$

soit d'après (1),

$$
-\operatorname{Ln}\left(\frac{1}{n}\right)=\left(\frac{h_{0}}{a}\right)^{c}=y_{0}
$$

Explicitant $p(h)$ et en posant $\left(\frac{h}{a}\right)^{c}=y$, il vient :

$$
\begin{aligned}
\text { н } 1 / n & =\frac{a \int_{y_{0}}^{\infty} e^{-y} y^{1 / c} d y}{\int_{y_{0}}^{\infty} e^{-y} d y} \\
\text { soit } \quad \text { H } 1 / n & =\frac{a}{e^{-y}} \int_{y_{0}}^{\infty} e^{-y} y^{1 / c} d y
\end{aligned}
$$

Ainsi, $\mathrm{H}(1 / \mathrm{n}) / \mathrm{H}(1 / \mathrm{m})$ pour deux valeurs distinctes de $\mathrm{n}$ et $\mathrm{m}$ sont indépendantes du paramètre " $\mathrm{a}$ ".

Une cascade de trois intégrations successives par partie, puis un développement en série intégrế terme à terme permettent d'estimer l'intégrale $I_{n}=\int_{z_{0}}^{\infty} z^{1 / c} e^{-z} d z$ par la relation :

$$
\begin{aligned}
& I_{n}=I_{0}+K\left[\frac{1}{\frac{1}{c}-3} z^{1 / c-3}-\frac{z^{1 / c-2}}{1 !\left(\frac{1}{c}-2\right)}+\cdots+(-1)^{n} \frac{z^{1 / c-n+3}}{n !\left(\frac{1}{c}+n-3\right)}\right]_{z_{0}}^{K} \\
& I_{0}=e^{-z_{0}} z_{0}^{1 / c}\left[1+\frac{z_{0}^{-1}}{c}+\frac{1-c}{c^{2}} z_{0}^{-2}+\frac{(1-c)(1-2 c)}{c^{3}} z_{0}^{-3}\right] \\
& \text { ct } \\
& K=\frac{(1-c)(1-2 c)(1-3 c)}{c^{4}}
\end{aligned}
$$

Pratiquerient il suffit de choisir $\dot{M}=6$ pour obtenir une bonnc estimation. 
Le tableau ci-dessous prêsente les valeurs de H(1/10)/a et $\mathrm{H}(1 / 3)$ a en fonction du paramètre de forme " $\mathrm{c}$ ", ainsi que les valeurs de $\bar{H} / a$ déduites des valeurs de $\Gamma\left(1+\frac{1}{c}\right)$.

\begin{tabular}{|c|c|c|c|c|c|c|c|}
\hline c & $\underline{\underline{\underline{R}}(1 / 1 / 10)} \frac{1}{2}$ & $\frac{y(1 / 3)}{a}$ & $\frac{\pi}{2}$ & c & $\frac{H(1 / 110)}{a}$ & $\frac{11(1 / 3)}{a}$ & $\frac{\pi}{a}$ \\
\hline 2 & 1.7999 & 1.4157 & 0.8862 & 5.5 & 1.2357 & 1.1291 & 0.9237 \\
\hline 2.5 & 1.5979 & 1.3160 & 0.8872 & 6 & 1.2140 & 1.1175 & 0.9270 \\
\hline 3 & 1.4767 & 1.2567 & 0.8934 & 6.5 & 1.1959 & 1.1079 & 0.9330 \\
\hline 3.5 & 1.3560 & 1.2133 & 0.9007 & 7 & 1.1807 & 1.0596 & $0.9360^{\circ}$ \\
\hline 4 & 1.3386 & 1.1835 & 0.9064 & 7.5 & 1.1676 & 1.0926 & 0.9350 \\
\hline 4.5 & 1.2956 & 1.1609 & 0.9137 & 8 & 1.1564 & 1. $480 \%$ & $0.9 \div 10$ \\
\hline 5 & 1.2623 & 1.1433 & 0.9182 & 8.5 & 1.1465 & 1.0811 & $0.9 / 35$ \\
\hline
\end{tabular}

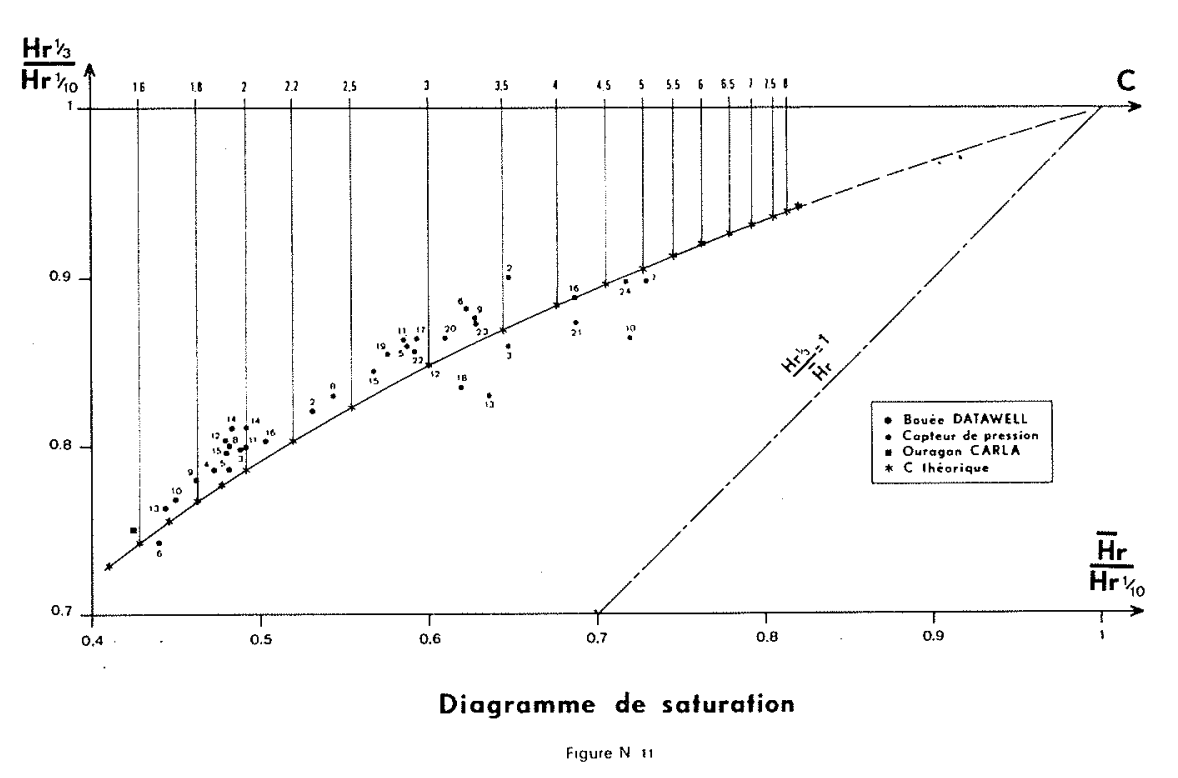

Figure $N$ :1

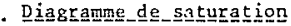

Le diagramne de saturation a pour système d'axe :

$\mathrm{H}(1 / 3) / \mathrm{H}(1 / 10)$ et $\overline{\mathrm{H}} / \mathrm{H}(1 / 10)$

Dans ce plan les couples de points $\mathrm{H}(1 / 3) / \mathrm{H}(1 / 10)$ et $\overline{\mathrm{H}} / \mathrm{H}(1 / 1 \mathrm{C}$

se situeront dans le triangle :

$0 \leqq H(1 / 3) / H(1 / 10) \leqq 1$

$0 \leqq \bar{B} / H(1 / 10) \leqq H(1 / 3) / H(1 / 10)$

Si 1 'on reporte sur un tel diagramme à la fois les points cor respondant aux différentes valeurs de "c" déduites du tableau ci-dessus et les points expérimentaux obtenus par le capteur de pression et la bouée Datawe1l (fig. 11) on constate la concordance satisfaisante du nuage de points avec la courbe thénrique, notamment aux fortes valeurs de " c ". La dispersion observée pour les valeurs du paramëtre

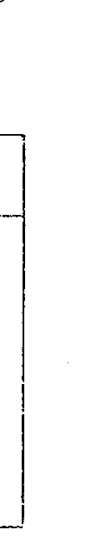




\section{CONCLUSTON}

Les distributions de gauss et de RAYLEIGH sont en toute rigueur valables pour représenter les variations de la surface libre tet la répartition deshauteurs de vagues si 1 'on admet que ces mouvements résultent d'une superposition d'ondes êlémentaires de fréquence voișine.

Au large, ces deux lois fournissent une bonne estimation des mouvements de la surface 1 ibre, bien que les hypothẽses théoriques ne soient pas toutes vếrifiées. Dans le cas de la houle côtiêre à la 1 imite du dēferlement les phénomènes non-1 inéaires deviennent rapidement prépondérants ; il en découle une forte dissyngtrie pour la rapartition du niveav et un ef fot de saturation pour les hauteurs de vagues dont on peut tenir compte en utilisant une distribution de WEIBULL dont la loi de RAMLEIGH est un cas particulier.

Par contre, pour 1 'estimation des courants particulaires, la théorie linéaire d'AIRY conduit à des résul tats sensiblement identiques à ceux déduits de la thêorie non-linéaire de l'onde longue, et très prochesdes résultats expérimentaux.

Ces résultats simples, obtenus à partir de mesures en site naturel permettent de présager favorablement de l'étape suivante qui doit aboutir à un raccordement entre les conditions au large et dans la zone de déferlement

par un modèle statistique.

\section{BIBLIOGRAPHIE}

[1] ARHAN M. et GOURITEN Y. (1974) - Relations entre les variations de pression au fond et les courants particulaires dans la houle côtière proche du déferlement. Contribution $\mathbb{N}^{\circ} 317$ du Département Scientifique du Centre Océariologique de Bretagne.

[2] BENDAT et PIERSOL (1971) - Rañdom Data. Wiley-Interscience.

[3] CARTUR IGHT et LONGUET-HIGGINS (1956) - The statistical distribution of the maxima of a random fonction. Proc. Roy. Soc. A 237 (1209) 212-231.

[4] CAVANIE A. et EZRATY R. (1974) - Etude statistique de la houle 1ittorale proche du déferlement. Contribution $\mathbb{N}^{0} 319$ du Dêpartement Scientifique du Centre Océanologique de Bretagne.

[5] GUMBEL E.J. (1958) - Statistics of extrenes. Columbia University Press.

[6] KINSMAN B. (1965) - Wind waves, their generation and propagation on the ocean surface. Prentice Hall.

[7] LONGUET-HIGGINGS M.S. (1952) - On the statistical distribution of the height of sea waves. J. Mar. Res. XI, $\mathrm{N}^{\circ} 3$, décerber 1952, PP. 245-266.

[8] LONGUET-HIGGINGS (1963) - The effects of non linearities on statistical distribution in the theory of sea-waves. Jour. Fluid Mech., Vol. 17, PP. $459-480$.

[9] LUKASIK Ṡ.J. et GROSCH C.E. (1963) - Pressure velocity correlations in ocean swell. Jour. Geoph. Res., Vol. 68, N²0, PP. 5689-5699.

[10] STOKER J.J. (1957) - Water waves. Interscience Publishers Inc., New-York.

[11] THOKPSON E.F. (1974) - Results from the C.E.R.C. measurement progran. Occan waves Measurements and Analysis. Waves 74. Vo1.1, International Symposium, New-Orleans, 9-11 september 1974.

[12] THORNTON E.B. et KRAPOHL R.F. (1974) - Water particle velocities measured under ocean waves. Jour. Geoph. Res., Vol.79, N6, PP. 847-852.

[13] TICK L.J: (1959) - A non 1 inear random model of gravity waves I. Jour. Math. Mech., Vo1. 8, PP. 643-652. 